\title{
Dermatitis in sheep in Mosul area
}

\author{
S.O. Youkhana \\ Coll.of Vet. Med./Unive. of Mosul
}

\begin{abstract}
Dermatitis in (25) sheep showing different distribution of the lesions were classified pathologically to acute, subacute, and chronic dermatitis. Grossly, the lesions were characterized by alopecia, erythema, exudation, ulceration, scale and crust formation. Histopathological sections revealed degeneration, spongiosis, intercellular edema with diffuse leukocytic infiltration predominantly neutrophils, lymphocytes and eosinophils in the dermal layer of the skin. The lesions were more progressive in chronic dermatitis.
\end{abstract}

\section{Introduction}

Dermatitis reaction is a non specific cutaneous inflammatory response with concomitant changes in the epidermis and dermis (1). The skin is particularly vulnerable to external damage by physicochemical or microbiologic insults but maintains macroscopically normal appearance over a wide range of environmental conditions and heals quickly after mild physical damage $(1,2)$. The reaction of the skin with underlying structures and other body systems, and its expression of general physiologic states, makes it an indicator of many nutritional deficiencies (3). However, the development of contaneous lesions often depends on both local and systemic factors (1, 2, 3). Many skin diseases of food producing animals are of considerable economic significance. There has been a tremendous expansion in both the number of dermatologic diseases recognized and in the understanding of their causes and pathogenesis (4). Dermatitis may be classified according to the nature and duration of the inflammatory response with epidermal and dermal changes, or on the basis of the infectious agent $(1,2)$. The purpose of the present study was to determine the pathological changes in the epidermis and dermis of acute, subacute and chronic dermatitis in sheep.

\section{Materials and Methods}

Twenty five skin specimens showing dermatitis in adult Awasi sheep were collected from Mosul abattoir. Tissue samples were fixed directly in $10 \%$ neutral formaldehyde saline for 48 hours, routinely

processed and embbeded in paraffin wax, sectioned at 4 um thickness and stained with hematoxylin and eosin. Toludine blue stain was used when necessary (6).

\section{Results}

\section{Acute dermatitis.}

Ten skin lesions showing patchy alopecia in the ventral abdomen, inner aspects of the thigh and near the tail, were characterized grossly by subepidermal papules, erythema and exudation. Histopathological sections revealed hydropic degeneration of the dermis. The dermoepidermal junctions was obscured by spongiotic vesicles, spongiosis, intercellular edema, vascular dilatation with diffuse leukocytic infiltration predominantly neutrophils (Fig. 1). The epidermis shows varying degree of vascular degeneration.

\section{Subacute dermatitis:}

Eight sheep showing lesions on the outer aspects of their ears were characterized grossly by erthyema, edema and exudation with extensive ulcers formation. Surrounded by areas of hemorrhages with mild to moderate vesiculation (Fig.2). Histopathologically proliferative changes were seen in the epidermis with varying degree of hyperplasia and hyperkeratosis. The 
dermal layer was infiltrated by numerous inflammatory cells mainly lymphocytes and eosinophils.

\section{Chronic dermatitis:}

Seven sheep showing lesions on the nose, face and around the eyes, were characterized by erythema, scaling and crust formation (Fig.3). Histopatho- logically the main epidermal changes were hyperplasia, hyperkeratosis, acanthosis and focal areas of spongiosis. Mild edema, vascular dilatation togather with inflammatory cells infiltration predominantly eosinophils accompanied by mast cells were the most prominent changes seen in the dermis (Fig. 4).

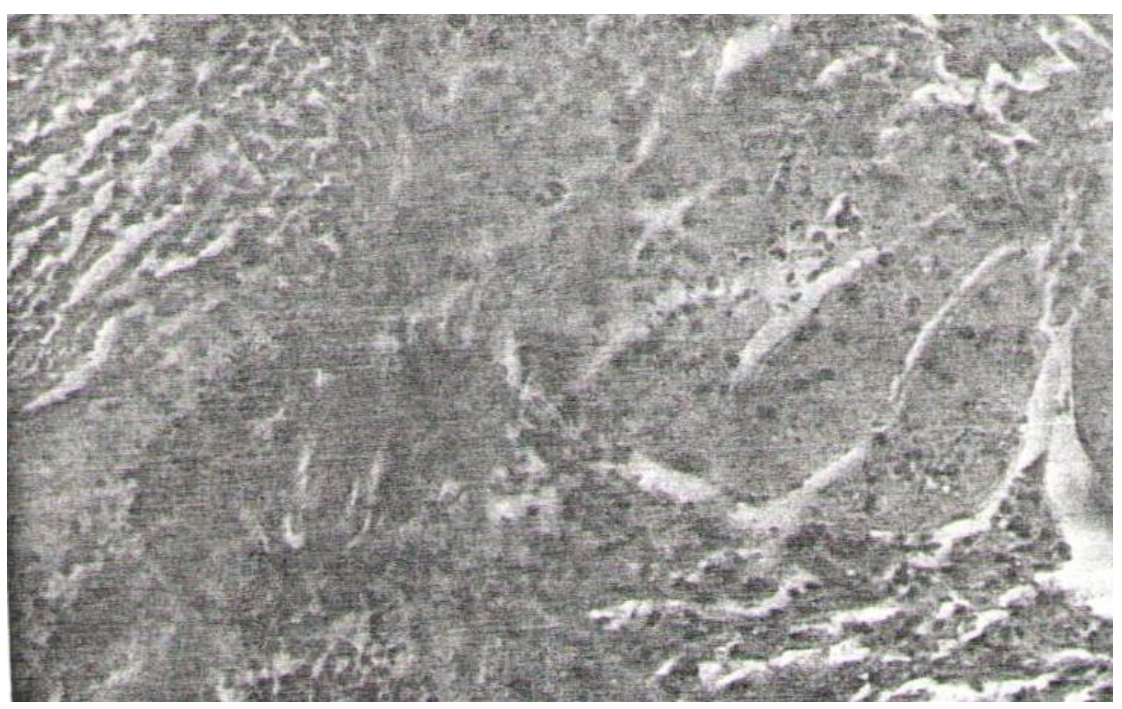

Fig.(1):Diffuse leukocytic infiltration predominantly neutropils the dermoepidermal junction in acute dermatitis. $\mathrm{H} \& \mathrm{E} 375 \mathrm{X}$.

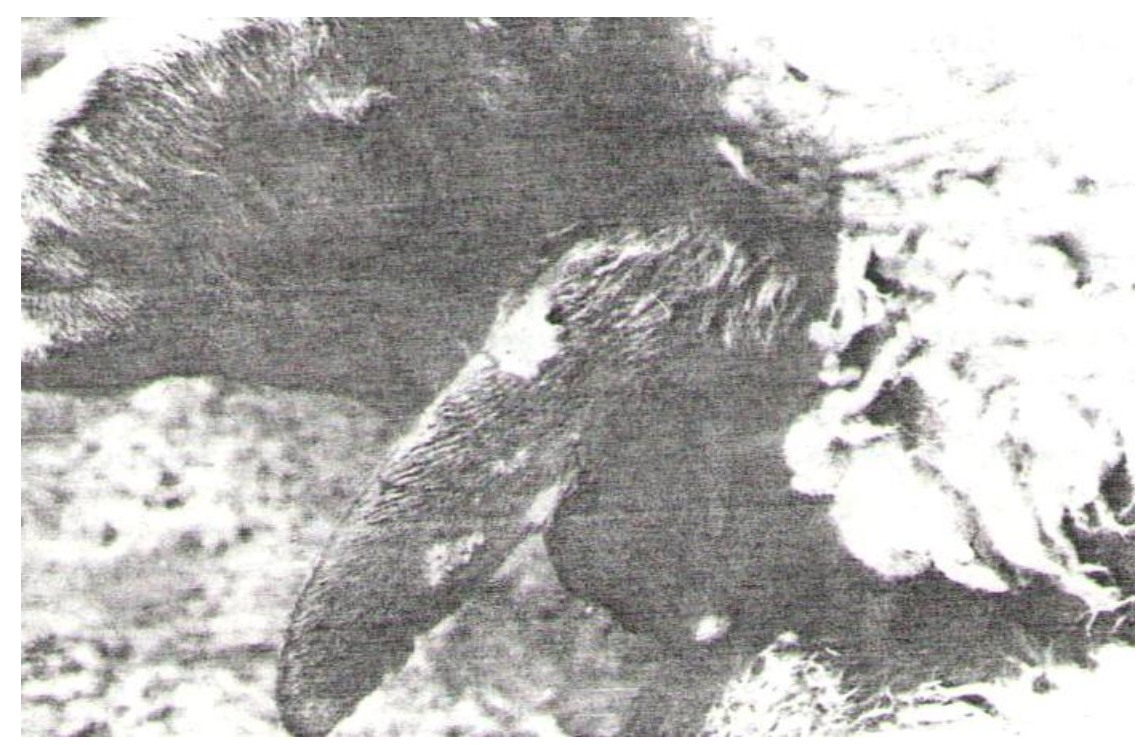

Fig. (2): Outer aspects of the ear showing ulcers surrounded by areas of hemorrhage with vesiculation in subacute dermatitis 


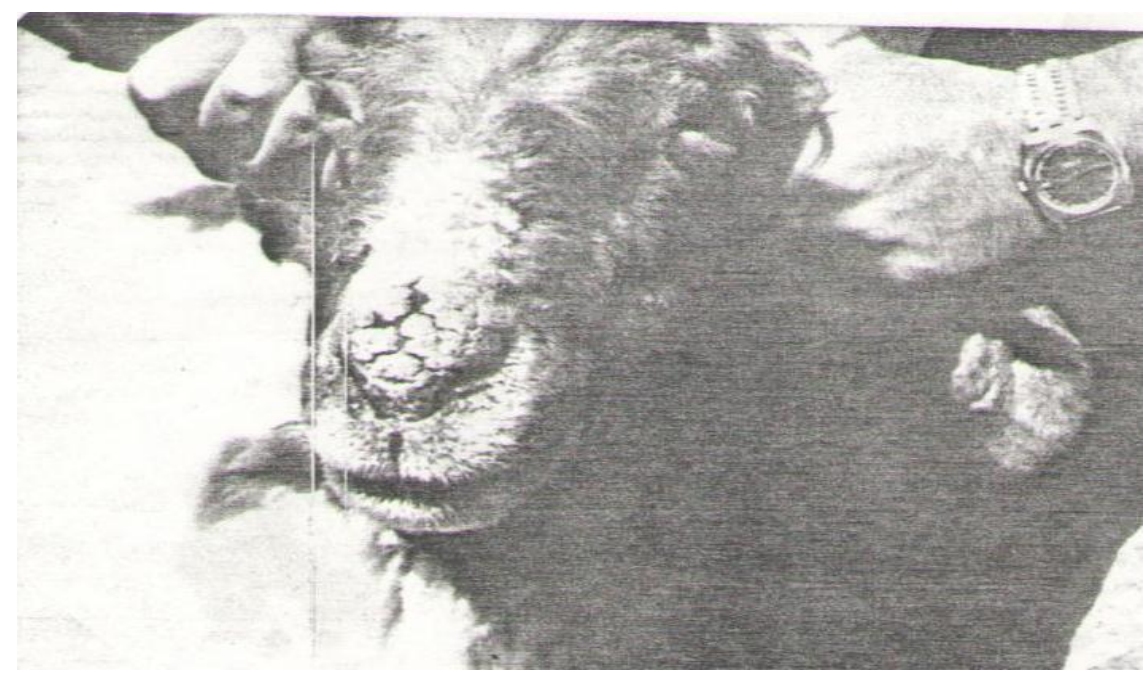

Fig(3): Upper aspect of a nose showing scales and crust formation in chronic dermatitis.

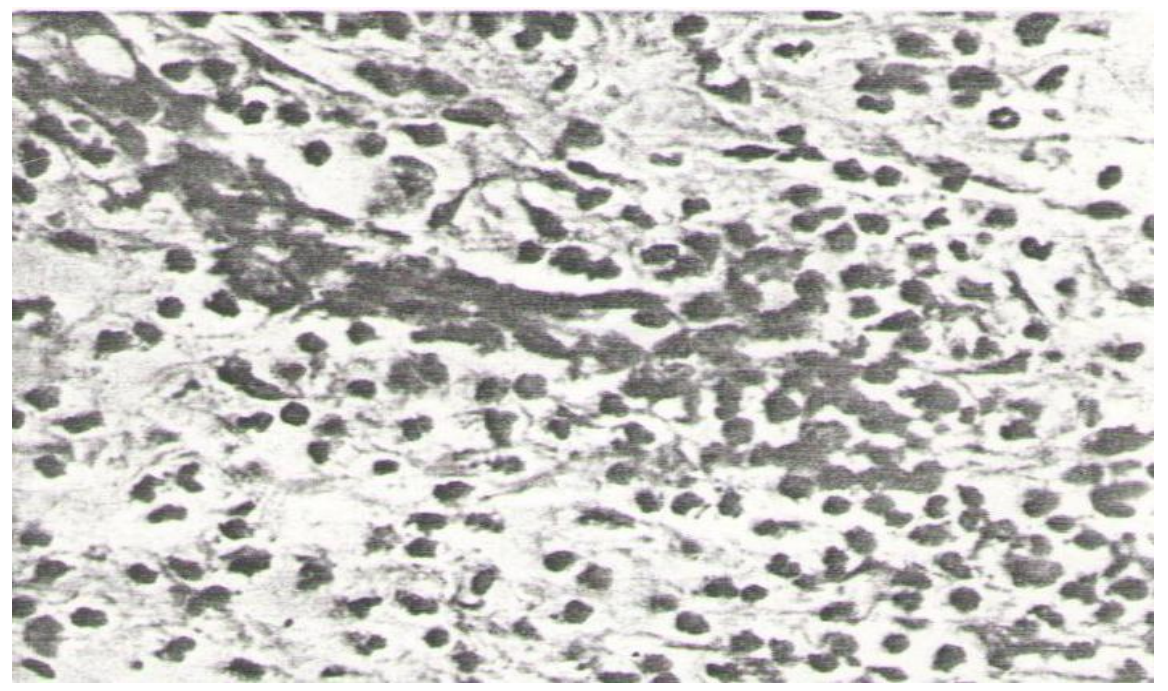

Fig.(4): Intercellular edema with eosinophil and mast cell infiltration in the dermis in chronic dermatitis.. H \& E 400 X.

\section{Discussion}

Dermatitis is oftenly associated with biting flies, and has been described in many parts of the world and in several mammalian species $(7,8,9,10,11)$. It occurs mainly in warmer months and affects the dorsal area of the body, especially the withers and base of the tail. Dermatitis was generally accepted to be an immediate hypersensitivity response (8, 10). Lesions in our study has a very different distribution occurring mainly on the ventral abdomen, inner aspects of the thigh, near the tail, outer aspects of the ears, nose, face and around the eyes. Grossly revealed patchy alopecia, erythema, exudation with extensive ulcer formation surrounded by areas of hemorrhage. In chronic cases scaling and crust formation were seen. Histopathologically, in acute and subacute dermatitis the lesions were distinctive to the epidermal and more deep to include the dermal layer. Epidermal and dermal inflammation was seen in all lesions. The most obscure changes were hydropic degeneration, vascular dilatation, 
intercellular edema and infiltration of neutrophils, lymphocytes and eosinophils. Proliferative changes were seen in epidermis. In chronic dermatitis the epidermal lesions were progressed to spongiosis, hyperkeratosis, and acanthosis associated with eosinophils and mast cell infiltration. These gross and histopathological changes seen in epidermis and dermis may be caused by trauma induced by purities, or it may be due to immediate hypersensitivity induced by flea bites (8).Further studies were needed to classify dermatitis of sheep in association with etiology of specific diseases specially those of the skin including external parasites, so that the pathological picture of the skin lesions may be more useful than the broad and etiologically nonspecific classification system of dermatitis.

\section{References}

1. Jubb KVF, Kennedy PC, Palmer, N. Pathology of domestic animals. $3^{\text {rd }}$. ed. New York, Academic Press, 1985; (1): 418-421.

2. Blood DC, Henderson JA, Radostits OM. Veterinary Medicine. $7^{\text {th }} \mathrm{ed}$. Baillere, Tindall, London, 1989; 483-484.

3. Martin WB. Disease of sheep. Blacwell Scientific Publication, Oxford, 1983; 196.

4. Jennings S. Some aspects of veterinary dermatology. Vet Rec. 1953; 65: 809-816.

5. Muller CH, Kirk RW, Scott DW. Small animal dermatology. $3^{\text {rd }}$ ed. Philadelphia : WB Saunders Co. 1983; 686-687.

6. Luna HC. Manual of histologic staining methods of the Armed Forces Institute of Pathology. $3^{\text {rd }}$ ed. MeGraw - Hill Company, 1968; 32-46.

7. Thelma LG, Kenneth WK, Gail AK. Correlation of histologic and immunologic findings in cats with milary dermatitis. J Am Vet Med Ass, 1986; 189 (10): 13221325.

8. Connan RM, Lioyd S. Seasonal allergic dermatitis in sheep. Vet Rec, 1988; 123 (13); 335-337.

9. Doherly ML, Bassett HF. Staphylococcal dermatitis in sheep. Vet Rec, 1989; 124 (17): 470.

10. Nimmo WJ, Yager JA, Nation PN, Clark EG, Townsend HG, Baird JD. Chrnoic eosinohpilic dermatitis : A manifestation of a Multisystemic, Eosinophilic, Epitheliotropic Disease in five horses. Vet Pathol, 1985; 22: 297-305.

11. Coignoul FL, Bertram TA, Martineau GP. Pathology of an ulcerative dermatitis in Belgian Landrace Sow. Vet Pathol, 1985; 22: 306310 .

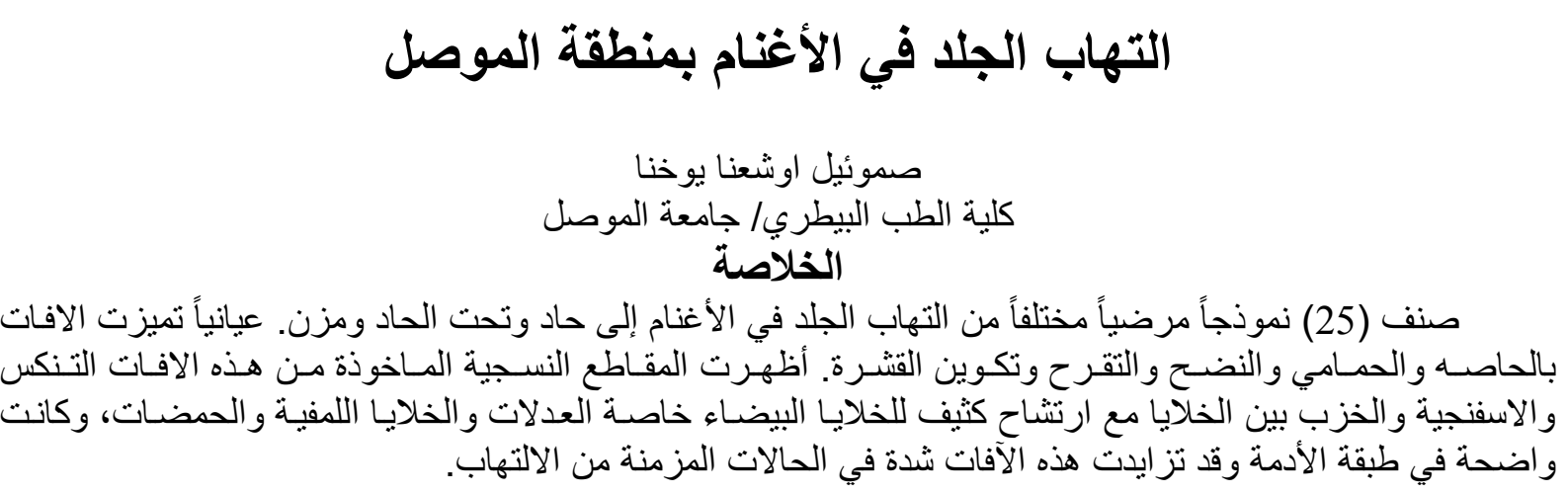

